\title{
Simultaneous Determination of Moisture, Protein and Fat in Fish Meal Using Near-Infrared Spectroscopy
}

\author{
Hui-Zhen ZHANG, ${ }^{1}$ Wei ZENG, ${ }^{1}$ Max RUTMAN ${ }^{2}$ and Tung-Ching LEE ${ }^{1 *}$ \\ 'Department of Food Science, the Center for Advanced Food Technology, and the Institute of Marine and Coastal Sciences, Rutgers University, \\ 65 Dudley Road, New Brunswick, New Jersey 08901-8520, USA \\ ${ }^{2}$ TPUAL S.A., General Ekdhal 159, Casilla 9649, Chile
}

Received March 2, 1999; Accepted October 4, 1999

\begin{abstract}
The near-infrared (NIR) spectrum of fish meal was determined on a scanning spectrophotometer. The multiple linear regression (MLR) and partial least squares (PLS) regression were used for the calibration of moisture, protein and fat contents in fish meal. Both the MLR and PLS gave high correlation coefficient and low standard error of calibration. Correlation coefficients of calibration were more than 0.90 for each component. The prediction results also show high correlation coefficients (more than 0.90 ). The relative errors for prediction of each component were less than $9 \%$, and for most samples were less than $6 \%$. Therefore, NIR spectroscopy could be a useful technique for simultaneously determining moisture, protein and fat contents in fish meal. The time required for analysis of one sample is less than $3 \mathrm{~min}$. It is a rapid and nondestructive method without environmental pollution. Thus, it will result in considerable time-saving, and it can be applied to on-line analysis at fish meal processing sites.
\end{abstract}

Keywords: fish meal, moisture, protein, fat, near-infrared spectroscopy

World landing of fish and shellfish is approaching 100 million metric tons (MMT) annually. Of this total, around $28 \%$ is processed into fish meal and oil (Bimbo \& Crowther, 1992). Fish meal is used in feed for poultry, pigs, ruminants and fish because it provides a unique balance of essential amino acids, energy, vitamins, and minerals which complement other feed ingredients by correcting any nutrition deficiencies (Pike, 1991).

To be able to control and optimize the processing of fish meal, it is important to measure and analyze its chemical composition. Traditionally, the fat content in fish meal was determined by organic solvent (e.g., acetone, ethyl ether or ethyl acetate) extraction for at least $16 \mathrm{~h}$. (AOAC, 1984), and the protein content in the fish meal was determined by a Kjeldahl procedure (AOAC, 1984), which must digest the sample using chemical reagents. The moisture content in the fish meal was determined by oven drying at a certain temperature for a certain period of time. Use of this standard method, however, is not only time consuming when a large number of samples must be analyzed, but also requires the use of a large quantity of chemical reagents, especially organic solvents. The chemicals, especially the volatile organic solvents are very harmful to an operator and cause environmental pollution. Nowadays, scientists are very aware of the environmental pollution (of water and air) by chemicals, especially volatile organic solvents. The treatment of wastewater polluted by chemicals, recovery of organic solvent and the prevention of solvent evaporation into the atmosphere pose formidable challenges. Therefore, it is imperative that a simple analytical method be developed for the determination of protein, fat, moisture contents in fish meal which is safe to both the operator and the environment.

*To whom correspondence should be addressed.

E-mail: lee@aesop.rutgers.edu
Near-infrared (NIR) spectroscopy analysis is a method for rapidly and reproducibly measuring the chemical composition of a sample with little or no sample preparation. Since NIR spectroscopic analysis offers four principal advantages: speed, simplicity of sample preparation, multiplicity of analyses from a single spectrum, and the intrinsic nonconsumption of the sample (McClure, 1994), it has been widely used to measure constituents of many agricultural commodities and food products as well as online analysis at food processing sites (Robert, 1987). The analyses of protein, oil, moisture and starch in agricultural commodities and food products were made easy, rapid and nondestructive. Osborne and Fearn (1986) determined the protein, fat and moisture in bread by NIR reflectance. Gjerde and Martens (1987) determined that NIR can be used to determine fat, moisture and protein in freeze-dried rainbow trout. Kim and Williams (1990) reported the determination of starch and energy in feed grains by NIR reflectance spectroscopy. Mitsumoto et al. (1991) evaluated NIR spectroscopy using reflectance, transmittance and fiber optic modes as a means of identifying physical and chemical characteristics of beef. Rasco et al. (1991) utilized NIR spectroscopy to estimate the proximate composition of trout muscle; Sollid and Solberg (1992) measured homogenized Atlantic salmon paste by diffuse NIR transmittance spectroscopy. Lee et al. (1992) used a short-wavelength NIR spectroscopic method to estimate the crude lipid content in the muscle of intact rainbow trout, and Isaksson et al. (1995) used NIR diffuse spectroscopy for the nondestructive determination of fat, moisture and protein in salmon fillets.

However, no technique is without some disadvantages and the principal one in the case of NIR is that it is empirical. There is no mathematical law which describes the interaction of radiation with a scattering medium containing a heterogeneous distribution of absorbing species. Therefore, the instrument readings are arbi- 
trary and require calibration using samples of known composition. As a further consequence of the empirical nature of NIR analysis, it is unwise to use calibrations to analyze sample types which were not included in the calibration set. Thus, even though there are many reports on use of NIR to determine the chemical compositions, we have rarely found reports where NIR spectroscopy has been used to determine the protein, moisture or fat content in fish meal. If the NIR technique can estimate the chemical compositions with direct reflectance mode from raw fish meal, it would result in considerable time-saving and minimize the chemical pollution resulting from traditional standard methods.

The objective of our study was to investigate the feasibility of the use of a NIR scanning instrument $(1100-2500 \mathrm{~nm})$ in the reflectance mode to determine moisture, protein and fat content simultaneously in fish meal with minimal sample pretreatment.

\section{Materials and Methods}

Materials Fish meal samples were commercial products that were made in Chile. Eighty-eight samples from different lots were used for preparation of calibration set and prediction set: 59 were randomly chosen as the calibration set, and 29 for the prediction set. The fish meal sample did not need any pre-preparation. It was ready for NIR analysis.

Chemical analyses Moisture content in fish meal samples was measured by oven drying at $100^{\circ} \mathrm{C}$ for $16 \mathrm{~h}$. Protein content was estimated from nitrogen content using the Kjeldahl technique (AOAC 18.026, 1984). Fat content was determined by ethyl ether extraction (AOAC 18.046, 1984).

NIR spectroscopy analysis All NIR measurements were made with a Model 6500 Spectrophotometer (Foss NIR Systems, Inc., Silver Springs, MD). This instrument contains a computer-based system with a single-scanning monochromator. The monochromator scans the range $1100-2500 \mathrm{~nm}$ in reflectance mode. Data were recorded at $2-\mathrm{nm}$ intervals and 32 scans were averaged for every sample. An empty standard, round sample holder was used as a reference. The lead sulfide detector was used for reflectance measurements. Spectral processing was performed on an IBM-386 interactive computer system. Regression computations were performed with an NSAS statistical software system (Foss NIR Systems, Inc.).

Mathematical treatment of raw NIR data Mathematical treatment modifies raw NIR spectral data. The treatment can eliminate the noise, correct the baseline variation, and enhance spectral data. Application of a mathematical treatment will prepare the raw spectral data for use in a regression and subsequent development of a calibration equation.

Smoothing NIR quantitative information can best be obtained when the data file has been smoothed. This smoothing process (e.g. N-point) is basically a means of averaging out the electro/ optical noise of the system so that the results are dependent only on true optical information, not on various noise spikes that could occur in the data.

In our study there were 11 wavelengths to be smoothed. This means that the optical data of the five wavelengths preceding a given wavelength is added to the optical data at that given wavelength. In addition, the optical information of the five wavelengths following the given wavelength is also added to this sum. This additive total is then divided by the smoothing divisor of 11 . The first and the last five wavelengths in the data file will not be properly averaged since there are no optical values preceding or following this data point.

Derivatives Derivatives are an approach to addressing two of the basic problems with NIR spectra: overlapping peaks and large baseline variations. The common method of calculating derivatives is finite-difference (Williams \& Norris, 1987).

If $\mathrm{A}$ is a spectrum defined for evenly spaced wavelengths $\lambda_{n}$, $n=0,1, \ldots, N-1$, then the first derivative $A_{n}^{\prime}$ at point $n$ is defined by:

$$
A_{n}^{\prime}=A_{n+g}-A_{n-g}
$$

where $g$ is an integer called the gap or derivative size and $A_{n}$ is the NIR value at point $n$. Similarly, the second derivatives are defined by:

$$
A_{n}^{\prime \prime}=A_{n+2 g}-2 A_{n}+A_{n-2 g} .
$$

Regression methods for calibration Calibration of the NIR instruments involved the establishment of a mathematical relationship between NIR response and the standard or reference method results. We use two multivariate procedures to perform the calibration: multiple linear regression (MLR) and partial least squares (PLS) regression,

MLR According to the Lambert-Beer Law, the general mathematical model of multi-component near-infrared reflectance is expressed as follows:

$$
Y=X_{i} E_{i}+e(i=1,2, \ldots, M),
$$

where $Y$ is the reflectance/absorbance vector of mixtures at different wavelengths. $E_{i}$ is the reflectivity/absorptivity vector of possibly present components $i$ at different wavelengths, and $e$ is the error vector which is a Gaussian distribution error of zero mean and uniform variance. $X_{i}$ is the concentration of component $i$ in the mixture and $M$ is the number of possibly present components.

If the components in all samples that will be measured are known and are the same, the $E_{i}$ can be determined from measurements of the reflectance spectra of the constituents present. From $E_{i}$ and $Y$ of the unknown sample, the concentration of constituents can be determined according to the following equation:

$$
X=Y E^{\mathrm{t}}\left(E E^{\mathrm{t}}\right)^{-1} \text {. }
$$

But in near-infrared, this kind of calibration (direct calibration) is complicated by strong interference from the noise factor caused by light scatter and unknown constituents.

Furthermore, some constituents do not have the same reflectance spectra in biological tissue as in their pure state. Therefore, the determination of constituents of complicated samples (such as food products) is difficult when using the direct calibration method.

We can rewrite equation (2) as

$$
\begin{gathered}
X_{i j}=Y_{i k} B_{k j} . \\
(i=1,2, \ldots, L ; j=1,2, \ldots, M ; k=1,2, \ldots, N)
\end{gathered}
$$

( $L$ : number of samples; $M$ : number of components in samples; $N$ : number of wavelengths)

$B$ is the coefficient of calibration. In equation (3), the $B$ can be estimated statistically from a set of representative calibration samples with measured chemical and near-infrared data. From $B_{j}$ (obtained from the calibration set) and $Y_{i}$ (NIR data of the unknown sample), the $X_{j}$ can be calculated easily. This method is called the indirect calibration method (Williams \& Norris, 1987). It is clear that this algorithm can be used in the analysis of a complicated sample.

PLS regression PLS regression method (Martens \& Naes, 
1991 ) is a factor-based procedure which can process full spectra. It factors the spectral data calibration matrix into the product of two smaller matrices. This involves a data compression step where the intensities measured at all the wavelengths used in the analysis are compressed to a small number of intensities in a new full-spectrum coordinate system. This system is composed of loading vectors that can be used to represent the original spectral data. A model is then applied to the intensities in the new fullspectrum coordinate system, called scores, that assumes the concentration to be a linear function of such intensities. The PLS method performs spectral factoring in order to account for the spectral variation while assuring that the new basis vectors relate to the calibration concentrations:

$$
\begin{aligned}
& X=F_{x} L_{x}+E_{x}, \\
& Y=F_{y} L_{y}+E_{y},
\end{aligned}
$$

where $X$ is the concentration matrix, $Y$ the analytical data matrix, $F_{x}$ and $F_{y}$ the score matrices, $L_{x}$ and $L_{y}$ the loading matrices, and $E_{x}$ and $E_{y}$ the residual matrices. The score matrices are related by

$$
F_{x}=F_{y} V+E_{c},
$$

where $V$ is the internal relationship and $E_{c}$ the residual matrix.

The unknown sample is quantified by determining $x_{0}$ and $y_{0}$ from

$$
x_{0}=y_{0}\left(F_{x}^{\mathrm{t}} Y\right)^{\mathrm{t}} V L_{x} \text {. }
$$

The calibration equation should give a high correlation coefficient of calibration $\left(R_{c}\right)$, a low standard error of calibration (SEC) and a low bias. The standard error of calibration measures how well the instrument matches calibration samples. Once the calibration equation is found, the prediction samples are used to verify the equation. The correlation coefficient of prediction $\left(R_{p}\right)$ and standard error of prediction (SEP) show how well the calibration can be used for the analysis of the samples.

\section{Results and Discussion}

Mathematical treatments for raw NIR spectral data Basically, the mathematical treatment (smoothing, first or second derivative) can correct the baseline, enhance spectral data or assist in smoothing a spectrum. Once the mathematical treatment has been applied, the information is prepared for use in calibration. In the present investigation, we use N-point smoothing, first derivative and second derivative mathematical treatments for modification of the NIR spectra and comparison with each other
(Tables 1 and 2).

MLR for calibration of moisture, protein and fat A major step in using MLR to obtain optimal results was the appropriate selection of the wavelengths to be used. The wavelengths selected by a stepforward-stepreverse regression for the three kinds of mathematical treatments in this study to provide the calibration equations with the lowest SEC and highest correlation coefficients of calibration $\left(R_{\mathrm{c}}\right)$ are given in Table 1 . The SEP and correlation coefficients of prediction $\left(R_{\mathrm{p}}\right)$ using these calibration equations for prediction are also shown.

From Table 1, we can see that the $R_{\mathrm{c}}$ obtained from second derivative mathematical treatment of spectra for moisture and protein are 0.97 and 0.96 , respectively, from N-point smoothing are 0.95 and 0.94 , respectively, and from first derivative are 0.96 and 0.95 , respectively. Therefore, the second derivative mathematical treatment of the spectra for moisture and protein was found to provide a better correlation than $\mathrm{N}$-point smoothing and first derivative. Also, the SECs obtained from second derivative mathematical treatment of spectra for moisture and protein are lower than $\mathrm{N}$-point smoothing and first derivative. However, the highest $R_{\mathrm{c}}$ and lowest SEC for fat were obtained from N-point smoothing mathematical treatment of the spectra.

After we obtained the calibration equations from the three mathematical treatments for each component in fish meal, we used them for prediction. From Table 1 , we can see that the $R_{\mathrm{p}}$ and SEP for moisture from the three calibration equations obtained by the three kinds of mathematical treatments are almost the same, but the $R_{\mathrm{p}}$ and SEP for protein and fat from the three calibration equations are much different from their $R_{\mathrm{c}}$ and SEC. The calibration equation obtained from the second derivative was found to give the highest $R_{\mathrm{p}}$ and lowest SEP. Although N-point smoothing gave the best $R_{\mathrm{c}}$ for fat, the $R_{\mathrm{p}}(0.82)$ is very low compared with the second derivative $\left(R_{\mathrm{p}} 0.94\right)$.

Therefore, using MLR to obtain the calibration equation and then to predict protein, fat and moisture contents in fish meal, second derivative mathematical treatment for the raw NIR spectra achieves better results than other mathematical treatments.

$P L S$ regression for calibration of moisture, protein and fat PLS is different from MLR. Generally speaking, PLS is useful with a small number of samples that contain some experimental

\begin{tabular}{|c|c|c|c|c|c|c|c|c|c|c|c|c|c|c|c|}
\hline Component & $\begin{array}{c}\text { Math. } \\
\text { treatment }\end{array}$ & $K_{0}^{(a)}$ & $K_{1}^{a t}$ & $K_{2}^{a)}$ & $K_{3}^{a)}$ & $K_{4}^{a)}$ & $\lambda_{1}^{{ }^{b)}}$ & $\begin{array}{c}\lambda_{2}^{\left({ }^{\prime \prime}\right.} \\
(\mathrm{nm})\end{array}$ & $\lambda_{3}{ }^{h \prime}$ & $\lambda_{4}{ }^{b)}$ & $R_{\mathrm{c}}^{\mathrm{c}}$ & $\mathrm{SEC}^{d)}$ & $\begin{array}{c}\text { SEP }^{\prime \prime} \\
(\%)\end{array}$ & $\begin{array}{l}\text { Bias } \\
(\%)\end{array}$ & $\begin{array}{l}R_{\mathrm{p}}^{f)} \\
(\%)\end{array}$ \\
\hline \multirow[t]{3}{*}{ Moisture } & $\mathrm{N}$-point & 10.22 & 244.35 & -9.84 & -189.91 & -46.14 & 1938 & 2238 & 1952 & 2414 & 0.95 & 0.28 & 0.29 & 0.014 & 0.93 \\
\hline & 1st derivative & 7.80 & 531.58 & -152.21 & -1229.27 & -313.95 & 1852 & 2030 & 2174 & 2060 & 0.96 & 0.25 & 0.30 & -0.029 & 0.93 \\
\hline & 2 nd derivative & 4.69 & -1678.34 & 177.34 & 706.60 & 893.23 & 1468 & 1718 & 2438 & 1822 & 0.97 & 0.22 & 0.31 & 0.0069 & 0.92 \\
\hline \multirow[t]{3}{*}{ Protein } & $\mathrm{N}$-point & 63.87 & 1088.29 & -275.00 & -1011.77 & 202.22 & 2298 & 1964 & 2310 & 1922 & 0.94 & 0.62 & 0.69 & 0.031 & 0.91 \\
\hline & 1st derivative & 69.89 & 590.70 & -710.85 & 711.54 & 1343.63 & 2024 & 2304 & 2234 & 2182 & 0.95 & 0.59 & 0.64 & -0.035 & 0.92 \\
\hline & 2nd derivative & 71.39 & -1908.14 & 1291.18 & -639.44 & -755.95 & 1628 & 1962 & 2254 & 2366 & 0.96 & 0.52 & 0.56 & 0.0064 & 0.94 \\
\hline \multirow[t]{3}{*}{ Fat } & $\mathrm{N}$-point & 13.67 & -3398.19 & 268.04 & -122.88 & 3245.30 & 2024 & 2396 & 2462 & 2206 & 0.99 & 0.12 & 0.52 & 0.0051 & 0.82 \\
\hline & 1st derivative & 9.61 & -728.78 & 576.39 & 247.12 & 202.39 & 1728 & 2306 & 1296 & 1938 & 0.93 & 0.29 & 0.34 & 0.035 & 0.93 \\
\hline & 2nd derivative & 10.77 & 877.32 & 254.98 & 360.27 & -201.96 & 1680 & 2174 & 2294 & 1718 & 0.95 & 0.24 & 0.32 & 0.071 & 0.94 \\
\hline
\end{tabular}
noise in the NIR. Therefore,further study of the use of PLS is

Table 1. Wavelengths selected and statistical summary of calibration and prediction for moisture, protein and fat in fish meal by near-infrared reflectance analysis using multiple linear regression.

"Constant: $K_{0}$ and regression coefficients: $K_{1-4}$ in the multiple regression model.

${ }^{b}$ Selected wavelengths.

Correlation coefficient of calibration.

${ }^{d)}$ Standard error of the calibration samples $(n=59)$.

'Standard error of the prediction samples $(n=29)$.

${ }^{n}$ Correlation coefficient of prediction. 
worthwhile for the calibration of moisture, protein and fat contents in fish meal.

Table 2 shows the statistical analysis of PLS using two differ- ent ranges of wavelength and three different mathematical treatments. PLS can be used for the full range of wavelength regression; however, selecting the wavelengths to be used for quan-

Table 2. Statistical summary of calibration and prediction for moisture, protein and fat in fish meal by NIR reflectance analysis using partial least squares regression.

\begin{tabular}{|c|c|c|c|c|c|c|c|c|c|}
\hline Component & Wavelength range $(\mathrm{nm})$ & Math. treatment & $\begin{array}{l}\text { No. of } \\
\text { factor }\end{array}$ & $\begin{array}{c}\operatorname{MSECV}^{a)} \\
(\%)\end{array}$ & $\begin{array}{l}\mathrm{SEC}^{b)} \\
(\%)\end{array}$ & $R_{\mathrm{c}}^{c)}$ & $\begin{array}{c}\mathrm{SEP}^{d)} \\
(\%)\end{array}$ & $\begin{array}{l}\text { Bias } \\
(\%)\end{array}$ & $R_{\mathrm{p}}{ }^{\prime \prime}$ \\
\hline \multirow[t]{6}{*}{ Moisture } & \multirow[t]{3}{*}{$1100-2500$} & $\mathrm{~N}$-point & 7 & 0.385 & 0.186 & 0.98 & 0.242 & -0.0258 & 0.95 \\
\hline & & 1st derivative & 4 & 0.596 & 0.303 & 0.95 & 0.308 & -0.0188 & 0.92 \\
\hline & & 2 nd derivative & 5 & 0.529 & 0.239 & 0.97 & 0.238 & -0.00506 & 0.95 \\
\hline & \multirow[t]{3}{*}{$1468-2438$} & $\mathrm{~N}$-point & 6 & 0.331 & 0.192 & 0.98 & 0.251 & -0.00858 & 0.95 \\
\hline & & 1st derivative & 4 & 0.510 & 0.274 & 0.96 & 0.288 & 0.00323 & 0.93 \\
\hline & & 2 nd derivative & 5 & 0.527 & 0.242 & 0.97 & 0.231 & -0.00748 & 0.96 \\
\hline \multirow[t]{6}{*}{ Protein } & \multirow[t]{3}{*}{$1100-2500$} & $\mathrm{~N}$-point & 6 & 1.771 & 0.541 & 0.96 & 0.624 & -0.0241 & 0.93 \\
\hline & & 1st derivative & 5 & 1.354 & 0.513 & 0.96 & 0.575 & -0.0591 & 0.94 \\
\hline & & 2 nd derivative & 5 & 1.446 & 0.495 & 0.97 & 0.595 & -0.0603 & 0.93 \\
\hline & \multirow[t]{3}{*}{$1628-2366$} & $\mathrm{~N}$-point & 7 & 1.337 & 0.407 & 0.98 & 0.593 & 0.0490 & 0.93 \\
\hline & & 1st derivative & 5 & 1.801 & 0.521 & 0.96 & 0.603 & -0.0616 & 0.93 \\
\hline & & 2nd derivative & 4 & 1.849 & 0.639 & 0.94 & 0.708 & -0.0239 & 0.90 \\
\hline \multirow[t]{6}{*}{ Fat } & \multirow[t]{3}{*}{$1100-2500$} & $\mathrm{~N}$-point & 6 & 0.558 & 0.304 & 0.93 & 0.402 & 0.0145 & 0.90 \\
\hline & & 1st derivative & 5 & 0.415 & 0.277 & 0.94 & 0.348 & 0.0576 & 0.92 \\
\hline & & 2 nd derivative & 3 & 0.565 & 0.321 & 0.91 & 0.449 & 0.0646 & 0.87 \\
\hline & \multirow{3}{*}{$1296-2462$} & $\mathrm{~N}$-point & 6 & 0.551 & 0.290 & 0.94 & 0.380 & 0.0208 & 0.91 \\
\hline & & 1st derivative & 5 & 0.483 & 0.284 & 0.94 & 0.345 & 0.0659 & 0.92 \\
\hline & & 2 nd derivative & 3 & 0.566 & 0.322 & 0.91 & 0.447 & 0.0661 & 0.87 \\
\hline
\end{tabular}

${ }^{a}$ Mean square error of cross validation.

b) Standard error of the calibration samples $(n=59)$.

c) Correlation coefficient of calibration.

d) Standard error of the prediction samples $(n=29)$.

e) Correlation coefficient of prediction.

Table 3. NIR prediction results using the calibration equation obtained by Partial Least Squares regression for moisture, protein and fat for fish meal, The wavelength range is from $1100 \mathrm{~nm}$ to $2500 \mathrm{~nm}$

\begin{tabular}{|c|c|c|c|c|c|c|c|c|c|}
\hline \multirow{2}{*}{$\begin{array}{c}\text { Sample } \\
\text { No. }\end{array}$} & \multicolumn{3}{|c|}{ Moisture content (\%) } & \multicolumn{3}{|c|}{ Protein content $(\%)$} & \multicolumn{3}{|c|}{ Fat content $(\%)$} \\
\hline & Chem method & NIR method & $\operatorname{RE}^{a}(\%)$ & Chem. method & NIR method & $\mathrm{RE}(\%)$ & Chem method & NIR method & $\mathrm{RE}(\%)$ \\
\hline 1 & 8.20 & 8.37 & 2.1 & 67.30 & 67.25 & 0.1 & 8.70 & 9.14 & 4.9 \\
\hline 2 & 9.60 & 9.11 & 5.2 & 66.00 & 65.19 & 1.2 & 8.50 & 9.27 & 8.6 \\
\hline 3 & 8.90 & 9.06 & 1.8 & 68.40 & 69.05 & 0.9 & 8.10 & 8.03 & 0.9 \\
\hline 4 & 8.80 & 8.62 & 2.0 & 68.60 & 69.25 & 0.9 & 8.80 & 8.05 & 8.9 \\
\hline 5 & 9.40 & 9.07 & 3.6 & 67.20 & 67.66 & 0.7 & 9.10 & 9.25 & 1.6 \\
\hline 6 & 7.90 & 7.89 & 0.1 & 70.00 & 70.22 & 0.3 & 8.10 & 8.17 & 0.9 \\
\hline 7 & 8.40 & 8.08 & 3.8 & 69.00 & 69.78 & 1.1 & 8.00 & 8.10 & 1.3 \\
\hline 8 & 9.30 & 9.53 & 2.5 & 68.50 & 66.83 & 2.5 & 8.80 & 8.62 & 2.1 \\
\hline 9 & 8.80 & 8.35 & 5.3 & 69.50 & 70.20 & 1.0 & 8.80 & 8.93 & 1.4 \\
\hline 10 & 9.70 & 9.56 & 1.5 & 67.20 & 67.83 & 0.9 & 9.00 & 9.21 & 2.3 \\
\hline 11 & 9.50 & 8.89 & 6.7 & 66.20 & 67.10 & 1.3 & 10.2 & 9.95 & 2.5 \\
\hline 12 & 10.0 & 9.96 & 0.4 & 68.20 & 68.58 & 0.6 & 8.50 & 8.00 & 6.0 \\
\hline 13 & 9.00 & 8.72 & 3.2 & 65.70 & 65.14 & 0.8 & 8.70 & 9.86 & 12 \\
\hline 14 & 7.90 & 7.96 & 0.7 & 70.60 & 71.05 & 0.6 & 8.00 & 7.97 & 0.4 \\
\hline 15 & 8.80 & 8.45 & 4.0 & 69.10 & 69.85 & 1.1 & 8.90 & 8.95 & 0.6 \\
\hline 16 & $\begin{array}{l}0.00 \\
11.1\end{array}$ & 11.4 & 2.9 & 63.40 & 62.65 & 1.2 & 9.80 & 9.66 & 1.4 \\
\hline 17 & 9.30 & 9.03 & 3.0 & 67.80 & 69.20 & 2.0 & 8.50 & 8.90 & 4.6 \\
\hline 18 & 9.30 & 9.37 & 0.8 & 67.40 & 68.15 & 1.1 & 9.40 & 9.08 & 3.5 \\
\hline 19 & 9.30 & 9.44 & 1.5 & 68.50 & 67.65 & 1.2 & 7.90 & 8.26 & 4.5 \\
\hline 20 & 8.60 & 8.11 & 5.8 & 66.10 & 66.30 & 0.3 & 10.0 & 10.1 & 1.0 \\
\hline 21 & 8.50 & 8.21 & 3.5 & 66.60 & 66.13 & 0.7 & 8.70 & 9.41 & 7.9 \\
\hline 22 & 9.70 & 8.95 & 8.1 & 67.40 & 67.38 & $<0.1$ & 9.70 & 9.09 & 6.5 \\
\hline 23 & 9.50 & 9.09 & 4.4 & 67.20 & 67.48 & 0.4 & 9.10 & 8.61 & 5.5 \\
\hline 24 & 9.10 & 9.30 & 2.1 & 68.00 & 68.15 & 0.2 & 8.20 & 8.51 & 3.7 \\
\hline 25 & 8.70 & 8.73 & 0.3 & 67.10 & 67.14 & 0.1 & 9.60 & 9.60 & 0.0 \\
\hline 26 & 9.70 & 10.1 & 4.3 & 63.10 & 64.51 & 2.2 & 10.9 & 10.7 & 1.6 \\
\hline 27 & 9.40 & 8.48 & 6.2 & 67.00 & 67.75 & 1.1 & 9.70 & 9.14 & 6.0 \\
\hline 28 & 8.70 & 9.12 & 4.8 & 69.10 & 69.10 & 0.0 & 8.90 & 8.15 & 4.5 \\
\hline 29 & 10.2 & 9.95 & 2.5 & 66.60 & 67.08 & 0.7 & 9.20 & 9.53 & 3.5 \\
\hline
\end{tabular}

${ }^{a}$ Relative error $(\%)=\{(\mid$ Chem. Method-NIR Method $\mid) /[($ Chem. Method+NIR Method $) / 2]\} 100$. 
tification is helpful. This was done by eliminating those wavelengths that provided no information on the components. The full range of wavelength used is $1100-2500 \mathrm{~nm}$. Based on the automatically best wavelength selection using a stepforward-stepreverse regression, the selected ranges of wavelength for moisture, protein and fat are 1468-2438 nm, 1628-2366 nm and 1296$2462 \mathrm{~nm}$, respectively. Comparison of $R_{\mathrm{c}}$ and SEC (Table 2) of the full range of wavelength with selected wavelength for each component and corresponding mathematical treatment, we found the selection of wavelength less important than the mathematical treatment of the raw NIR spectra. The important factor affecting $R_{\mathrm{c}}$ and SEC of PLS for each component still is mathematical treatment of the raw NIR spectra.

From Table 2 we can see that the effects of different mathematical treatments of spectra are different from moisture, protein and fat. Their $R_{c}, \mathrm{SEC}$ and $R_{\mathrm{p}}, \mathrm{SEP}$ are shown in the table. For moisture, we found that N-point smoothing and second derivative give better $R_{c}, \mathrm{SEC}$ and $R_{\mathrm{p}}$, SEP than first derivative. For protein and fat, we found that N-point smoothing and first derivative give higher $R_{\mathrm{c}}, R_{\mathrm{p}}$ and lower $\mathrm{SEC}, \mathrm{SEP}$ than second derivative.

Therefore, we can use either wavelength range (full range of wavelength or selected wavelength) for PLS of fish meal to obtain the highest $R_{\mathrm{c}}$ and lowest SEC. To determine moisture content, we can use the $\mathrm{N}$-point smoothing and second derivative as mathematical treatments of raw NIR spectra. We can use the $\mathrm{N}$-point smoothing and first derivative as mathematical treatments of raw NIR spectra to determine protein and fat content.

Comparison of MLR and PLS regression for calibration of moisture, protein and fat in fish meal From Tables 1 and 2, we can see that the $R_{\mathrm{c}}, R_{\mathrm{p}}, \mathrm{SEC}$ and SEP of moisture, protein and fat content using MLR and PLS are not significantly different. Therefore, we can use any calibration equation obtained from either MLR or PLS for the prediction of these three contents in fish meal.

Using calibration equation for prediction We have discussed above that using any calibration equation obtained from either MLR or PLS for the prediction of moisture, protein and fat content in fish meal is acceptable. Thus, we use the prediction results from the calibration equation obtained by PLS as an example (Table 3). From Table 3, we can see that the relative errors between chemical method and NIR method for protein, fat and moisture content in fish meal are less than $9 \%$. For moisture content, most of the relative errors are less than $5 \%$; the relative errors of only six samples are in the range of $5-8.1 \%$. For protein, most relative errors are less than $1.5 \%$; those of only three samples are in the range of $2.0-2.5 \%$. For fat, most relative errors are less than $6.0 \%$; those of five samples are in the range of $6.5-$ $8.9 \%$.

Therefore, we have shown the NIR method can be used for the simultaneous determination of moisture, protein and fat content in fish meal. The relative errors for determination are acceptable.

\section{Conclusions}

Our developed NIR method reported here minimizes the environmental concerns associated with the use of organic solvents and hazardous reagents. The technique can simultaneously determine protein, moisture and fat contents in fish meal. The time for analysis of one sample was less than $3 \mathrm{~min}$. The relative errors for prediction of each component were less than $9 \%$, and those for most samples were less than $6 \%$. It is a rapid and nondestructive method and causes no environmental pollution. Thus, it will result in considerable time-saving and can be applied to on-line analysis at fish meal processing sites.

Acknowledgments This work was supported partially by Rutgers/NOAA Cooperative Marine Education and Research (CMER) Program and partially by State Funds and a Hatch project.

\section{References}

AOAC. (1984). "Official Methods of Analysis (14th Ed.)." Association of Official Analytical Chemists, Arlington, VA, USA.

Bimbo, A.P. and Crowther, J.B. (1992). Fish meal and oil: Current uses. J. Am. Oil Chem. Soc., 69, 221-227.

Gjerde, B. and Martens, H. (1987). Predicting carcass composition of rainbow trout by near-infrared reflectance spectroscopy. J. Anim. Breed Genet., 104, 137-148Tфgersin.

Isaksson, T., T $\phi$ gersen, G., Iversen, A. and Hildrum, K.I. (1995). Nondestructive determination of fat, moisture and protein in salmon fillets by use of near-infrared diffuse spectroscopy. J. Sci. Food Agric., 69, $95-100$.

Kim, H.O. and Williams, P.C. (1990). Determination of starch and energy in feed grains by near-infrared reflectance spectroscopy. $J$. Agric. Food Chem., 38, 682-688.

Lee, M.H., Cavinato, A.G., Mayes, D.M. and Rasco, B.A. (1992) Non-invasive short-wavelength near-infrared spectroscopic method to estimate the crude lipid content in the muscle of intact rainbow trout. J. Agric. Food Chem., 40, 2176-2181.

Martens, H. and Naes, T. (1991). "Multivariate Calibration." Wiley, New York, USA.

McClure, M.F. (1994). The giant is running strong. Anal. Chem., 66, 43A-53A.

Mitsumoto, M.. Maeda, S., Mitsuhashi, T. and Ozawa, S. (1991) Near-infrared spectroscopy determination of physical and chemical characteristics in beef cuts. J. Food Sci., 56, 1493-1496.

Osborne, B.G. and Fearn, T. (1986). "Near Infrared Spectroscopy in Food Analysis." Longman Scientific \& Technical, Longman House, Essex.

Pike, I.H. (1991). Increasing importance of aquaculture to the fish meal industry. International Association of Fish Meal Manufacturers, Technical Market Report, April.

Rasco, B.A., Miller, C.E. and King, T.L. (1991). Utilization of NIR spectroscopy to estimate the proximate composition of trout muscle with minimal sample pretreatment. J. Agric. Food Chem., 39, 6772 .

Robert, P., Bertrand, D., Devaux, M.F. and Grappin, R.M. (1987). Multivariate analysis applied to near-infrared spectra of milk. Anal. Chem., 59, 2187-2191.

Sollid, H. and Solberg, C. (1992). Salmon fat content estimation by near infrared transmission spectroscopy. J. Food Sci., 57, 792-793.

Williams, D.C. and Norris, K. (eds.) (1987). "Near-Infrared Technology in the Agricultural and Food Industries," American Association of Cereal Chemists, Inc., St. Paul, MN, USA. 